brevipetiolatis, superioribus sessilibus tri-quinque-fidis, summis parvis bracteiformibus oblongo-linearibus integerrimis; floribus $3-5$ in cymam terminatem capitatam dispositis, breviter pedunculatis, magnis; calycis lobis ovatis obtusissimis, petalis calyce duplo longioribus late obovatis, leviter emarginatis. trinerviis, albis, antheris luteis (?). Tota planta glanduloso- et viscido-pubescens, obscure virens.

Hab. in Lusitania centrali, ubi in agro Cintrano crescit. Floret Majo.

Diese ron Herrn Oberst v. Kuzinský aufgefumdene Art gehört zur Section Nephrophyllum, in welcher sie der von mir in den Illustrationes Florae Hispaniae insularumque Balearium (Band I. S. 7, t. VII) beschriebenen und abgebildeton $S$. blanca aus Aragonien am nächsten steht. Letztere unterschejdet sich aber von der Pflanze von Cintra durch ibre ungestielten länglichen eingeschnitten-gezähnten bis fiederspaltigen Blätter und die länglichen, việ schmäleren Blumenblätter.

\title{
Zwei neue hybride Orchideen.
}

Von R. v. Wettstein und G. Sennholz.

(Mit $\ddot{~ J l l u s t r a t i o n e n .) ~}$

1. Orchis speciosissima (Fig. 4 a-c).

(O. speciosa Host Fl. Austr. II, p. $527 \times 0$. sambucina I. Fl. Suec. p. 312.)

Tuberidia obscure lobata; radices adventitiae filiformes. Caulis validus, strictus, teres in parte superiore subangulosus, in parte inferiore foliosus, superiorem versus foliis sparsis obsitus. Folia inferiora obovato-lanceolata, superiora lanceolato-cuneata acuta, omnia glabra nitida, basin versus maculis purpureis adspersa. Spica densiuscula ovato-elongata, circa $5 \mathrm{~cm}$. longa. Bracteae inferiores flores superantes, superiores floribus aequilongae, lanceolatae, longe acuminatae, basi $3-4 \mathrm{~mm}$. latae, virides vel apicem versus purpurascentes, 3-5 nerviae, nervis inter se nervis secundariis connexis. Ovarinm modice tortum. Perigonii phylla externa (Fig. 4 a) oblonga, apicem versus longe acuminata, acuta, trinervia, lateralia patentia, 8-10 mm. longa; phylla interna (Fig. 4b) lateralia minora, ovato-lanceolata, obtusa, binervia vel subtrinervia. Labellum (Fig. 4c) breviter trilobum, lobis lateralibus breviter acuminatis, subdentatis, lobo medio emarginato, denticulato, basin versus subpapilloso. Calcar cylindraceum, obtusum, orario aequilongum, horizontale.

Inter parentes frequentissimos in pratis supra Klein-Zell (V, 1886, Wettstein) et inter locum Brennalpe dictum et cacumen 
montis Reisalpe Austriae inferioris (VI, 1889, Sennholz) utroque in loco unicum specimen.

Die Mittelstellung in allen Merkmalen, das ganz vereinzelte Vorkommen zwischen den beiden oben genannten Arten, O. speciosa Host und $O$. sambucina L., lässt es als ganz sicher erscheinen, dass die im Vorstehenden beschriebene Orchis eive Hybride darstellt. Dio Pflanze ist sehr auffallend, da sie die habituellen Eigenthümlichkeiten der beiden, sich so wenig gleichenden Stammarten vereint.

Von Orchis sambucina (Fig. 1) unterscheidet sich O. speciosissima durch die weniger getheilten Knollen, die etwas breiteren, gegen die Basis roth gefleckten Blätter, die längere, schmälere, zerstreut blüthige Aehre, die kürzeren Deckblätter, wagrechten Sporne, die Farbe der Blüthen und die Form der Blüthentheile, die aus Fig. 4 ersichtlich ist.

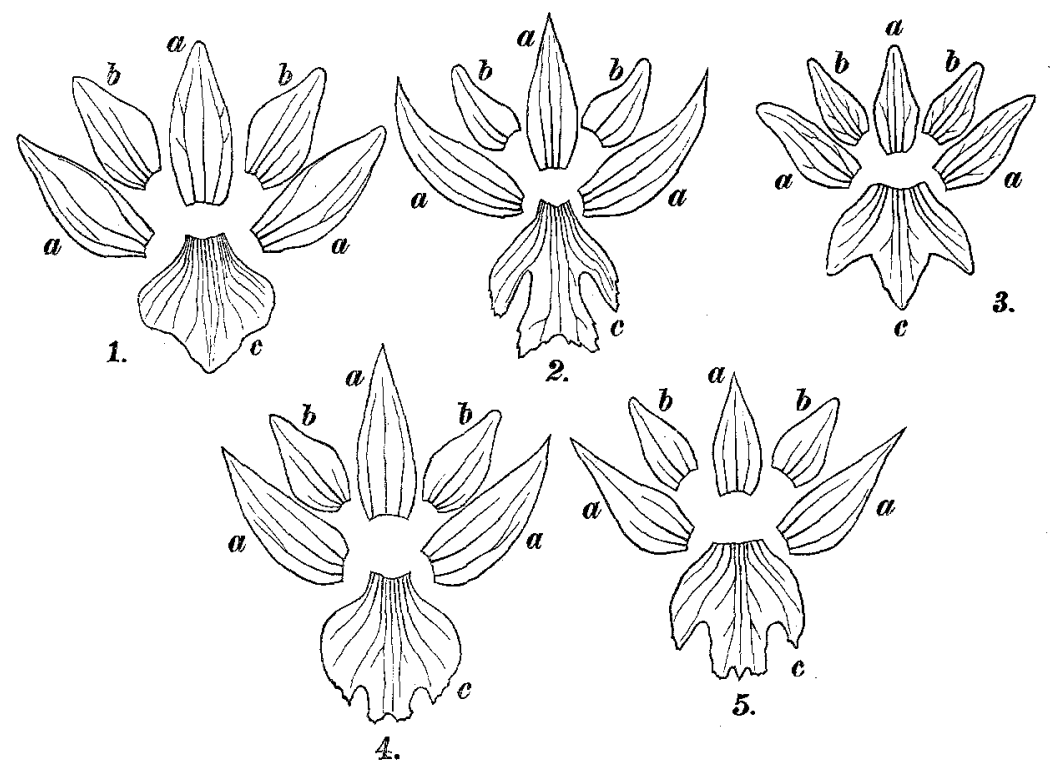

Fig. 1. Orachis sambucina L. -- 2. O. speciosa Host. - 3. O. maculata L. - 4. O speriosissima Sennh. et Wettst. - 5. O. Pentecostalis Sennh. et Wettst. In allen Figuren bedentet: a die ausseren, $b$ die inneren Perigonzipfel, $c$ das Labellum.

Die Form der Unterlippe ist gerade bei den beiden Stammarten von grosser Gleichförmigkeit, weshallb der Bastart daran um so leichter zu erkennen ist.

Von O. speciosa (Fig. 2) ist O. speciosissima durch schwach getheilte Knollen, höher hinauf beblätterten Stengel, weniger gefleckte, schmälere Blätter, kürzere und gedrungenere Aehren, längere Bracteen, die Form nnd Stellung des Sporns, die kürzeren, weniger spitzen Perigonzipfel und die weniger tief getheilte, mehr abgerundete Unterlippe verschieden. 
Da O. speciosa und $O$. sambucina in den niederösterreichischen Voralpen, so insbesondere im Gebiete zwischen der Reisalpe und dem Schneeberge sehr häufig in grosser Menge zusammen wachsed, dürfte die beschriebene Hybride hier noch mehrfach beobachtet werden können; das von Senuholz gesammelte Exemplar wird im botanischen Garten der Wiener Universität cultivirt.

\section{Orchis Pentecostalis (Fig. 5 a-c).}

(O. speciosu Host $\times$ maculata L. Spec. plant. p. 942.)

'luberidia obsolete palmata; radices adventitiae filiformes. Canles strieti, elati, subangulati, maculis nonnullis purpureis, in parte superiore foliis pancis lanceolatis acuminatis obsitus. Folia late lanceolata, obtusinscula, in pagina superiore maculis paucis purpureis basin versus minimis obsita, in pagina inferiore glaucoviridia. Spica elongata, densiuscula. Bracteae acuminatae, lanceolatae, inferiores floribus fere aequilongae, superiores breviores, trinerviae. Perigonii phylla externa oblonga acuta trinervia (Fig. 5a), lateralia patentia supremo longiores, phylla interna (Fig. 5 b) ovato-oblonga, obtusa, subtrinervia. Labellum (Fig. 5e) late cunoato-rotundatum, trilobum, lohis lateralibus brevibus acutis, lobo medio breviter trilobo denticulato. Calcar cylindraceum ovario subbrevius horizontale vel modice dependens. Flores pallide purpurascentes, phyllis exterioribus saturatius purpureis, labello maculato.

Specimen unicum iuter parentes in pratis inter locum Brennalpe dictum et cacumen montis Reisalpe Austriae inferioris (VI, 1889, Sennholz).

Diese im Habitus von beiden muthmasslichen Stammarten abweichende Pflanze nuterscheidet sich von ibnen durch folgende Merkmale: Von O. speciosa L. (Fig. 2) durch etwas getheilte Knollen, schmälere und relativ längere, stärker gefleckte Blätter, kürzere und dichtere Blüthenähren, kürzere und stumpfere Perigonzipfel, die Form des Labellums, die Blüthenfarbe, den wagrechten oder etwas nach abwärts gerichteten Sporn u. s. w.; von O. maculuta L. (Fig. 3) durch kürzere Blätter, schmälere, längere und weniger dichtbliuthige Aehren, spitzere äussere Perigonzipfel, deu zwei- bis dreilappigen gezähnten Mittelzipfel der Unterlippe, den nicht herabhängenden Sporn n. a. m.

Dis Auffindung einer Hybride zwischen $O$ speciosa Host und O. maculata L, gibt auch Gelegenheit, zum Theile Klarheit über eine andere Pflanze zu erbalte», nämlich über $O$. Spitzelii Saut. (Koch Synops. I. p. 686), die mehrfach für eine Hybride zwischen den beiden Arten, respective $\mathrm{z}$ wischen $O$. mascula L. und maculata L. gehalten wurde. (Vergl. Oest. bot. Zeitschr. 1876, S. 263). Halacsy hat bereits in den Nachtrïgen zur Flora von Nieder-Oesterreich (S. 58, 1882) neuerdings diese Ansicht für unberechtigt erklärt und zwar mit vollem Rechte, da die $O$. Spitzelii sich von $O$. Pentecostalis (wir verglichen von dieser Exemplare aus dem Val di 
Ledro und Monte Baldo) vor Allem schon durch die relativ kurzen, abgerundeten Blätter, die stumpfen Perigonzipfel, dio dreilappige Unterlippe mit stumpfen Zipfeln und die Farbe unterscheidet. - - Eine Hybride zwischen O. speciosa und maculata ist demnach O. Spitzelii gewiss nicht.

\section{Lepidotrichum Vel. Born.}

eine neue Cruciferengattung aus dem Gebiete der pontischen Flora.

\section{Von Dr. J. Velenovský.}

Im vorigen Jahre hat mein Freund I. Bornmüller, in dieser Zeitschrift eine ausführliche Beschreibung des Plilotrichum Uechtritzianum Born., einer nenen Crucifere von Varna, veröffentlicht. Diese interessante Pflanze habe ich im Jahre 1885 bei Varna auf dem sandigen Meerstrande unweit von Galata zum erstenmale gefunden, wo sie mit anderen interessanten Strandpflanzen recht häufig vorkommt. Als ich im Jahre 1886 meine ersten Beitiäge zur Flora ron Bulgarien publicirte, musste ich die Varnaer Crucifere unerwälınt lassen, weil ich nur blühende Exemplare besass, und aus dem Habitus der Pflanze nicht einmal annähernd die Gattung feststellen konnte. Die nächsten Ferien botanisirte I. Bor'nmüller in der Umgebung von Varna und sammelte nun die fragliche Crucifere in blühenden und fruchttragenden Stücken. Als ich von Bornm üller die Fruclitexemplare dieser Pflanze bekam, konnte ich auch diesmal noch nicht foststellen, in welche Gattung die Pflauze zu stellen sei. Dass man es hier mit einer nenen Art zu thun hat, war schon ganz sicher, und Bornmüller hat dieselbe a. a. 0 . als Ptilotrichum beschrieben, obwohl er mit Recht bemerkte, dass seine neue Pilotrichum-Art mit keiner Art dieser Gattung naho Verwand tschaft zeige.

Mit der systematischen Bearbeitung der Flora von Bulgarien befasst, gelangte ich in der letzten Zeit zu den Cruciferen und da habe ich das Pilotrichum Uechtritzianum nochmals einer genauen Untersuchung unterworfen. Eine allseitige Vergleichung mit allen bekannten Arten der Gattungen Alyssum, Koniga, Ptilotrichum und Berteroa führt mich nun zu der definitiven Ueberzengung, dass das nene Ptilotrichum eine selbststäudige, von den vier genannten weit verschiedene Gattung repräsentirt. Bornmüller hat mir in einer brieflichen Mittheilung aus Amasia fremudlichst gestattet, die betreffende Beschreibung zusammenzustellen und zu veröffentlichen.

Unsere Pflanze ist ansgesprochen ausdauernd und entwickelt mehrere, häufig niederliegende oder aufsteigende, verzweigte, rigide, dünne Stengel. Die Bläthentrauben sind kurz, gestreckt, seitlich und torminal. Die angedrückt silberglänzende Bekleidung besteht aus schuppenförmicen Steruhaaren. Habituell ähnelt die neue Pflanze am meisten dem Ptilotrichum longicaule Boiss. aus Granada, weniger 